DOI: https://doi.org/10.46296/gt.v4i8edesp.0033

\title{
MANIFESTACIONES DE ANSIEDAD EN DOCENTES DEBIDO A CONFINAMIENTO POR SARS-COV-2
}

\section{ANXIETY MANIFESTATIONS IN TEACHERS DUE TO SARS-COV-2 CONFINEMENT}

\author{
Monge-Cedeño Diana María ${ }^{1}$; García-Cedeño María Leonila ${ }^{2}$ \\ ${ }^{1}$ Estudiante de la Maestría Académica con Trayectoria de Investigación en Psicología, Mención \\ Psicoterapia. Instituto de Posgrado de la Universidad Técnica de Manabí, UTM. Portoviejo, \\ Ecuador. Correo: dmonge1046@utm.edu.ec. ORCID ID: https://orcid.org/0000-0003-3209-039X \\ ${ }^{2}$ Docente Investigador de la Universidad Técnica de Manabí, UTM. Portoviejo, Ecuador. \\ Correo: maria.garcia@utm.edu.ec. ORCID ID: https://orcid.org/0000-0003-0202-2193
}

\begin{abstract}
Resumen
Los docentes de la Unidad Educativa Fiscomisional Juan Montalvo, a raíz de la suspensión de clases presenciales por la pandemia del Sars- Cov-2, han continuado labores de manera virtual, y al igual que el resto de las personas, han tenido que afrontar acontecimientos vitales estresantes; tales como contagio por covid-19, pérdidas familiares y dificultades económicas. Situación que ha traído consigo la expresión de ciertas manifestaciones de angustia. El presente trabajo tiene como objetivo cuantificar los niveles de ansiedad en la población descrita mediante el uso del Inventario de Ansiedad de Beck (BAI) y una encuesta sobre datos demográficos; instrumentos aplicados a 16 docentes del subnivel básica elemental y media de la mencionada institución educativa. Se hizo uso de una investigación no experimental de tipo descriptiva, obteniendo como resultado que, pese a las diversas calamidades presentadas por la población docente durante la pandemia, se evidencia en un alto porcentaje niveles de ansiedad que oscilan entre la categoría normal y moderada y dentro de los rangos en mención, una leve prevalencia de síntomas subjetivos sobre los síntomas somáticos. Se concluye que la baja incidencia de niveles de ansiedad en los docentes podría responder a varios factores protectores grupales que se implementaron desde el inicio de la pandemia; sin embargo, se recomienda implementar otro tipo de instrumentos tales como entrevistas individuales y otras escalas que permita conocer la particularidad de la sintomatología en los sujetos de estudio.
\end{abstract}

Palabras clave: Ansiedad; Sars- Cov-2; docentes.

\begin{abstract}
The teachers of the Juan Montalvo Fiscomisional Educational Unit, as a result of the suspension of face-to-face classes due to the Sars-Cov-2 pandemic, have continued their work in a virtual way, and like the rest of the people, they have had to face events stressful vitals; such as contagion by covid-19, family losses and financial difficulties. Situation that has brought with it the expression of certain manifestations of anguish. The present work aims to quantify anxiety levels in the population described by using the Beck Anxiety Inventory (BAI) and a survey on demographic data; instruments applied to 16 teachers of the basic elementary and middle sublevel of the aforementioned educational institution. A descriptive non-experimental research was used, obtaining as a result that, despite the various calamities presented by the teaching population during the pandemic, a high percentage of anxiety levels that oscillate between the normal and moderate categories and within of the ranges mentioned, a slight prevalence of subjective symptoms over somatic symptoms. It is concluded that the low incidence of anxiety levels in teachers could respond to several group protective factors that were implemented since
\end{abstract}

Información del manuscrito:

Fecha de recepción: 13 de julio de 2021.

Fecha de aceptación: 27 de septiembre de 2021.

Fecha de publicación: 12 de noviembre de 2021. 
the beginning of the pandemic; However, it is recommended to implement other types of instruments such as individual interviews and other scales that allow knowing the particularity of the symptoms in the study subjects.

Keywords: Anxiety; Sars-Cov-2; teachers.

\section{Introducción}

EI SARS-CoV-2 no únicamente trajo consigo repercusiones a nivel de la salud física de quienes la padecieron, sino que, parte de la población mundial experimentó la sensación de temor ante una enfermedad desconocida que, inicialmente parecía no dar tregua y contra la cual, el mundo continúa luchando sin encontrar alguna respuesta totalmente satisfactoria, pese a la existencia en el mercado de varias vacunas que prometen alto nivel de efectividad.

Las pérdidas de familiares, el desempleo, las dificultades económicas, el miedo a lo desconocido, el exceso de información, la obligatoriedad de adaptación a nuevos espacios y el temor a contagiarse o a contagiar a los seres queridos, son situaciones ansiógenas que, sin lugar a duda, provocaron y provocan diversas reacciones en la población en general. Uno de los sectores en los que más se ha evidenciado los cambios en mención, ha sido el sector educativo. Acorde a la UNESCO, a nivel global, las actividades educativas de niños, niñas, adolescentes y adultos se han visto afectadas, por lo que, desde esta organización, se está brindado apoyo a varios países, con el objetivo de promover y facilitar la continuidad del aprendizaje (UNESCO, 2020).

Dentro de las diversas comunidades educativas, tanto estudiantes como docentes han sido afectados. Este último grupo se ha visto afectado de manera significativa ya que tuvieron que abandonar sus aulas y un elevado porcentaje de ellos, adaptarse a la modalidad virtual, la cual produjo diversas manifestaciones de preocupación y ansiedad.

En relación a lo antes expuesto, Gómez y Rodríguez (2020) refieren que, las disposiciones para que los docentes hagan uso de herramientas pedagógicas virtuales, trajo consigo ansiedad e incertidumbre, ya que su 
uso era muy poco frecuenta en la modalidad presencial y en la actualidad los docentes se han visto en la necesidad de aprender a utilizarlas.

Acorde a Diario el Universo, en una encuesta aplicada a 1200 docentes, el $22 \%$ dio a conocer que en algún momento durante el confinamiento se sintió tensionado, o presentó algún cambio de humor brusco. Así como también un porcentaje significativo expresó haber presentado dificultades para conciliar el sueño (EL UNIVERSO, 2020).

Situación similar ocurre en los docentes de la Educativa Fiscomisional Juan Montalvo, quienes, en su mayoría, son migrantes digitales y desde el inicio de la pandemia, emprendieron la ardua labor del aprendizaje del uso de las Tics, lo que, sin lugar a duda, sumado a las preocupaciones por otros aspectos de la pandemia, elevó sus niveles de temor, situación se evidenciaban en el desborde emocional que varios presentaban en las reuniones virtuales.

La presente investigación, tiene como objetivo cuantificar los niveles de ansiedad en los docentes de los subniveles básica elemental y media de la Unidad Educativa Fiscomisional Juan Montalvo de la ciudad de Manta. Mediante el desarrollo de esta, se contribuirá de manera significativa a los postulados y abordaje de la problemática existente. Cabe indicar que, se cuenta con los recursos requeridos para la ejecución de este estudio, tales como accesibilidad a elementos teóricos, predisposición de la población para participar, así como también la apertura en la unidad educativa donde se llevará a efecto el mismo.

\section{Metodología (Materiales y métodos)}

Se llevó a efecto una investigación no experimental, de tipo descriptivo en una población de veinte profesores que, acorde a la secretaría del Vicerrectorado de la Unidad Educativa Fiscomisional Juan Montalvo, pertenecen a la planta docente de la sección básica elemental y media a quienes se le aplicó de manera virtual el Inventario de Ansiedad de Beck (BAI) ,que ha demostrado validez en la detección de síntomas ansiosos(Padrós 
Blázquez et al., 2020) y al que se accede mediante el siguiente enlace: https://cutt.ly/1jNc47I . Se excluyó a aquellos docentes que, no hayan autorizado su participación y a los que, durante el proceso, no dieron respuesta a varios ítems del instrumento.

Dentro de los aspectos éticos, se hizo uso del consentimiento informado, así como también se tomaron en cuenta principios de rigor científico, la Declaración Universal de Derecho Humanos y principios de la bioética en investigación en Ciencia Sociales, por lo cual, no se divulgarán ningún dato que afecte a la integridad de los participantes. Finalmente, para el procesamiento de información estadística, se utilizó el Software SPSS y Microsoft Excel.

\section{Resultados (análisis e interpretación de los resultados)}

El $68.8 \%$ de la población investigada pertenecía al sexo femenino, y la edad prominente de los participantes fue de entre 30 y 40 años. Acorde al Inventario de Ansiedad de Beck, este grupo presentó niveles de ansiedad entre leve y moderado. En la población comprendida entre los 50 y 60 años, se presenta una variabilidad en cuanto a los niveles de ansiedad. $Y$ en el grupo de docentes cuya edad oscila entre los 20 y 30 años, se identificaron niveles normales.

Tabla 1. Correlación entre edad y niveles de ansiedad

\begin{tabular}{|c|c|c|c|c|c|c|}
\hline & & & $\begin{array}{c}\text { Leve a } \\
\text { Moderada }\end{array}$ & $\begin{array}{c}\text { Moderada a } \\
\text { Severa }\end{array}$ & Normal & \\
\hline \multirow[t]{8}{*}{ Edad } & \multirow{2}{*}{$\begin{array}{l}\text { Entre } 20 \text { y } 30 \\
\text { años }\end{array}$} & Recuento & 0 & 0 & 2 & 2 \\
\hline & & $\%$ del total & $0,0 \%$ & $0,0 \%$ & $12,5 \%$ & $12,5 \%$ \\
\hline & \multirow{2}{*}{$\begin{array}{l}\text { Entre } 30 \text { y } \\
40 \text { años }\end{array}$} & Recuento & 7 & 0 & 3 & 10 \\
\hline & & $\%$ del total & $43,8 \%$ & $0,0 \%$ & $18,8 \%$ & $62,5 \%$ \\
\hline & \multirow{2}{*}{$\begin{array}{l}\text { Entre } 40 \text { y } \\
50 \text { años }\end{array}$} & Recuento & 0 & 0 & 1 & 1 \\
\hline & & $\%$ del total & $0,0 \%$ & $0,0 \%$ & $6,3 \%$ & $6,3 \%$ \\
\hline & \multirow{2}{*}{$\begin{array}{l}\text { Entre } 50 \text { y } 60 \\
\text { años }\end{array}$} & Recuento & 1 & 1 & 1 & 3 \\
\hline & & $\%$ del total & $6,3 \%$ & $6,3 \%$ & $6,3 \%$ & $18,8 \%$ \\
\hline \multirow[t]{2}{*}{ Total } & & Recuento & 8 & 1 & 7 & 16 \\
\hline & & $\%$ del total & $50,0 \%$ & $6,3 \%$ & $43,8 \%$ & $100,0 \%$ \\
\hline
\end{tabular}

En cuanto a la subdivisión de la BAI, en las categorías de ansiedad subjetiva y ansiedad somática, se evidencia una mayor incidencia de niveles de ansiedad subjetiva en la población estudiada, en cuyos ítems 
se evidencia que en varias seleccionaron la opción ocasiones los participantes "Moderadamente".

Gráfico 1. Ansiedad subjetiva.

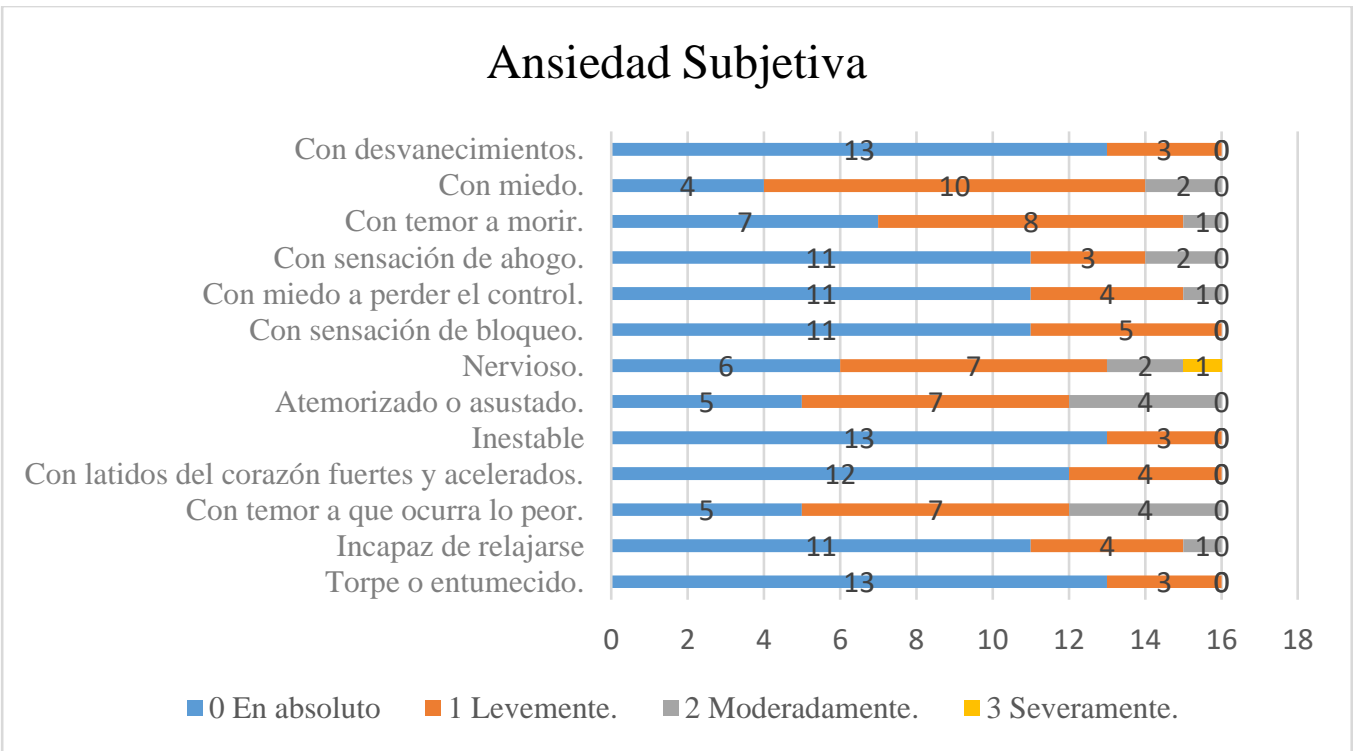

Gráfico 2. Ansiedad somática.

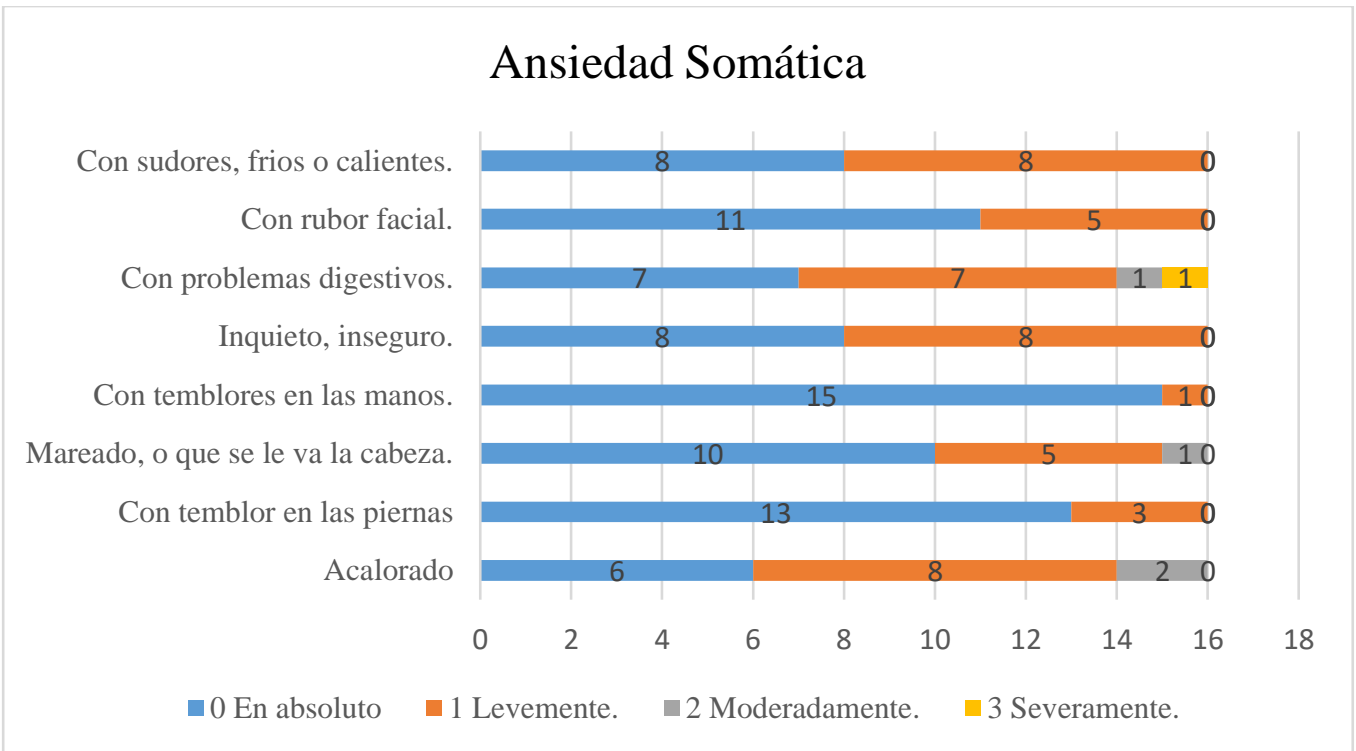

Por otra parte, no fue posible definir con precisión la incidencia de síntomas ansiosos por sexo, debido a que el $68.8 \%$ de la población investigada pertenecía al sexo femenino. Sin embargo; se identificó una leve prevalencia de sujetos masculinos dentro de la categoría de niveles de ansiedad normal. 
Tabla 2. Correlación entre niveles de ansiedad y sexo.

\begin{tabular}{|c|c|c|c|c|c|}
\hline & & $\begin{array}{l}\text { Leve a } \\
\text { Moderada }\end{array}$ & $\begin{array}{c}\text { Moderada a } \\
\text { Severa }\end{array}$ & Normal & \\
\hline \multirow{2}{*}{ Sexo } & Femenino & 7 & 1 & 3 & 11 \\
\hline & Masculino & 1 & 0 & 4 & 5 \\
\hline Total & & 8 & 1 & 7 & 16 \\
\hline
\end{tabular}

Fuente: Encuesta e Inventario de Ansiedad de Beck

\section{Discusión}

Desde el inicio de la pandemia, dentro de las comunidades educativas el mayor índice de medidas preventivas, han estado focalizadas en pro del bienestar de los estudiantes y de sus familias, relegando de alguna manera las necesidades del docente. Acorde a (Quispe \& García, Gianmarco, 2020), el colectivo docente, está inmerso dentro de la población afectada, ya que generalmente los profesores solían distribuir su tiempo entre las actividades del hogar y las inherentes a la docencia, pero, en la actualidad, ambas se realizan en el hogar. Acorde a (Román et al., 2020) "Maestros y profesores enfrentaron una situación inesperada y única. Para la gran mayoría, el aislamiento preventivo fue una experiencia nueva, lo cual significó un desafío que puso en juego las habilidades cognitivas, emocionales y sociales de todos".
En la población docente de la Unidad Educativa Fiscomisional Juan Montalvo pertenecientes a la sección básica elemental y media, desde el inicio de la pandemia, se empezaron a aplicar medidas que facilitaron la migración de la enseñanza desde la modalidad presencial a la virtual, tales como reuniones mediante la herramienta virtual Zoom, en donde los integrantes de la comunidad educativa dominaban el uso de herramientas virtuales enseñaban a aquellos que desconocían el mismo.

Este tipo de intervenciones, aparte de dotar a los docentes de conocimientos y estrategias sobre la enseñanza virtual, significó un espacio de catarsis ya que posterior a los encuentros, los docentes relataban diversas situaciones ansiógenas y el grupo, haciendo uso de una escucha empática, brindaba ciertas directrices de afrontamiento ante la situación. Se presume que esto, pudo haber incidido en el alto porcentaje de niveles de ansiedad 
normal $(43,8)$ leve y moderada (50\%), que dio como resultado la aplicación del Inventario de ansiedad de Beck en la población descrita. Ferrari et al.., citando a Gracia, expresan que las intervenciones grupales facilitan el desarrollo de la autonomía y permiten al sujeto satisfacer necesidades básicas afectivas, de seguridad y de autoestima, promoviendo de esta manera la acción social. (Ferrari et al., 2000)

Acorde al Manual Diagnóstico y Estadístico de Enfermedades Mentales, quinta edición (DSM-V) , la ansiedad prevalece en doble proporción en mujeres, en relación a los varones (APA, 2014). En el presente estudio, debido a que el $68.8 \%$, de la población era de sexo femenino, no fue posible precisar con exactitud este dato, pero se identificó una leve prevalencia de sujetos masculinos dentro de la categoría de niveles de ansiedad normal.

\section{Conclusiones}

Acorde a la aplicación del Inventario de Ansiedad de Beck (BAI), en los docentes del subnivel básica elemental y media de la Unidad
Educativa Fiscomisional Juan Montalvo, se presentan niveles de ansiedad que oscilan entre normal, leve y moderada con una leve preponderancia de síntomas subjetivos sobre los somáticos. La escasez de caso con niveles de ansiedad severa podría responder a una diversidad de encuentro virtuales grupales que funcionaron como espacios de escucha y contención durante el año lectivo 2020- 2021. Se recomienda implementar instrumentos tales como entrevistas individuales y otras escalas que permita conocer la particularidad de la sintomatología en los sujetos de estudio, así como también el uso de estrategias preventivas individuales y grupales.

\section{Bibliografía}

APA. (2014). Manual diagnóstico y estadístico de los trastornos mentales (V). Editorial Médica Panamericana.

EL UNIVERSO. (2020). En Ecuador, alumnos y docentes se sienten muy angustiados por pandemia del COVID-19; cuatro de cada diez, tensionados | Ecuador | Guayaquil | El Universo. https://www.eluniverso.com/g uayaquil/2020/10/12/nota/801 1690/salud-mental-covid-19- 
ecuador-alumnos-docentesangustiados-unicef

Ferrari, L. M., Gaiztarro, A. R., Lavín, G. M., \& Eisman, G. S. (2000). Las aportaciones de los grupos de autoayuda a la salud mental. CLÍNICA Y SALUD, 27.

Nidia Gómez Dávalos \& Pablina Rodríguez Fernández. (2020). ESTRÉS EN DOCENTES EN EL CONTEXTO DE LA PANDEMIA DE COVID-19 Y LA EDUCACION, FENOB UNA | AcademicDisclosure. https://revistascientificas.una. py/ojs/index.php/rfenob/article /view/150

Padrós Blázquez, F., Montoya Pérez, K. S., Bravo Calderón, M. A., \& Martínez Medina, M. P. (2020). Propiedades psicométricas del Inventario de Ansiedad de Beck (BAl, Beck Anxiety Inventory) en población general de México. Ansiedad y Estrés, 26(2), 181187.

https://doi.org/10.1016/j.anye s.2020.08.002

Quispe, F., \& García, Gianmarco. (2020). Impacto psicológico del covid-19 en la docencia de la Educación Básica Regular | Alpha Centauri. http://journalalphacentauri.co m/index.php/revista/article/vie $w / 10$

Román, F., Forés, A., Calandri, I., Gautreaux, R., Antúnez, A., Ordehi, D., Calle, L., Poenitz,
V., Pérez, K. L. C., Torresi, S., Barcelo, E., Conejo, M., Ponnet, V., \& Allegri, R. (2020). Resiliencia de docentes en distanciamiento social preventivo obligatorio durante la pandemia de COVID-19. Journal of Neuroeducation, 1(1), 76-87. https://doi.org/10.1344/joned. v1i1.31727

UNESCO. (2020). La educación en América Latina y el Caribe ante la COVID-19. UNESCO. https://es.unesco.org/fieldoffic e/santiago/covid-19education-alc 